\title{
Corrections to effect size variances for continuous outcomes of cross-over clinical trials
}

\author{
Barbara Kitchenham $^{\dagger}$ and Lech Madeyski* ${ }^{*}$ and François Curtin ${ }^{\S}$
}

Keywords: cross-over trials; continuous data; effect sizes; effect size variances; corrections

\section{Introduction}

We would like to make some corrections to the formulas presented in the 2002 Statistics in Medicine article by Curtin et al. [1]. That article presented formulas for the variances of standardized weighted mean difference of an AB/BA cross-over trial that would be comparable both with parallel designs and cross-over designs.

There are three main issues in the Curtin et al.'s paper [1] that we address in this communication. Firstly, the paper proposes a standardized effect size for cross-over trials that is inconsistent with the standardized effect sizes used for other repeated measures designs such as the pretest-posttest studies used in educational studies, see [2] and [3]. Secondly, the change to the standardized effect size for cross-over studies necessitates a change to variance of the standardized effect size. Thirdly, the variance of the standardized effect size comparable with parallel trials was not based on the distribution of a valid $t$-variable, so includes some errors. We follow Curtin et al.'s approach and base our revised variance equations on the moments of the non-central $\mathrm{t}$-distribution, replacing the $t$-variable with a variable based on the effect size.

\section{The standardized effect sizes from $A B / B A$ cross-over trials}

Using Curtin et al.'s notation, the original paper derived the expectation and variance of the standardized mean difference of the mean cross-over trial with a period effect from the equation:

$$
\frac{\bar{d}_{X O}}{s_{x}}=\frac{\frac{1}{2}\left(\bar{d}_{A B}+\bar{d}_{B A}\right)}{s_{x}}
$$

$\bar{d}_{A B}$ and $\bar{d}_{B A}$ are the mean cross-over differences in sequences $\mathrm{AB}$ and BA respectively and $s_{x}^{2}$ is the pooled within sequence cross-over difference variance.

However, if we want a standardized effect size for cross-over designs that is consistent with other repeated measures, the unstandardized effect size $\bar{d}_{X O}$ should be standardized by the within-subject standard deviation, $s_{e}$ [3]. $s_{e}$ is also a natural choice for the standardizer because $s_{e}^{2}$ is the random effects residual term obtained when analysing cross-over data with a linear mixed model. Since Curtin et al. note that $s_{x}^{2}=2 s_{e}^{2}$, it is easy to calculate $\frac{\bar{d}_{X O}}{s_{e}}$. For consistency with [3], we refer to this standardized effect size as $d_{R M}$, which is an estimate of the parameter $\delta_{R M}$.

Curtin et al. correctly propose basing a standardized effect size comparable with parallel trials on the within- plus between-subject variance, $\sigma_{b}^{2}$. For comparison with Morris and DeShon [3] we refer to this as $d_{I G}$, where $I G$ refers to

\footnotetext{
* Correspondence to: Lech Madeyski, Faculty of Computer Science and Management, Wroclaw University of Science and Technology, Wyb.Wyspianskiego 27, 50-370 Wroclaw, Poland. Email: Lech.Madeyski@pwredu.pl

${ }^{\dagger}$ School of Computing and Mathematics, Keele University, Keele, Staffordshire ST5 5BG, UK.

${ }^{\ddagger}$ Faculty of Computer Science and Management, Wroclaw University of Science and Technology, Wyb. Wyspianskiego 27, 50-370 Wroclaw, Poland.

${ }^{\S}$ Research Center for Statistics, Geneva School of Economics and Management, University of Geneva, Switzerland and Geneuro SA, Geneva, Switzerland
} 
independent groups, so:

$$
d_{I G}=\frac{\bar{d}_{X O}}{s_{b}}
$$

while $d_{I G}$ estimates the parameter $\delta_{I G}$. Curtin et al. point out that $\sigma_{b}^{2}=\sigma_{\xi}^{2}+\sigma_{e}^{2}$ where $\sigma_{\xi}^{2}$ is the between-subject variance.

Morris and DeShon [3] point out that $d_{R M}$ estimates the standardized expected change for individuals while $d_{I G}$ estimates the standardized expected difference between the two methods. They note that either viewpoint might be the objective of meta-analysis.

\section{Standardized effect size variances}

The variance estimate most suitable for small samples (up to $\approx 30$ participants) for any standardized mean difference effect size is derived from the non-central $t$ distribution [3, 2]. Furthermore, Johnson and Welch [4] report the variance of a $t$ variable with mean $\theta$ to be:

$$
V(\theta)=\frac{d f}{d f-2}\left(1+\theta^{2}\right)-\frac{\theta^{2}}{[c(d f)]^{2}}
$$

Where $\theta$ is estimated by the sample $t$-value and $d f=\left(n_{A B}+n_{B A}-2\right)$ is the degrees of freedom associated with the $t$-test. Hedges [5] provides exact values of $c(d f)$ for values of $d f$ up to 50. In addition, Morris [2] confirmed that $c(d f)$ is well-approximated by $\left(1-\frac{1}{4 d f-1}\right)$ even for small samples when estimating the variance of pretest-posttest standardized effect sizes.

If we know the relationship between $\theta$ and a standardized effect size $\delta$ is given by the equation:

$$
\theta=A \times \delta
$$

where $A$ is a constant term, then, the variance of $\delta$ is:

$$
\operatorname{var}(\delta)=\frac{1}{A^{2}} V(\theta)
$$

This is true for any standardized effect size that can be calculated from a $t$-value, including those obtained from repeated measures crossover designs, repeated measures pretest-posttest designs, and independent group designs.

In the case of a cross-over design, Senn [6] points out that the $t$ test is based on:

$$
t=\frac{2 \bar{d}_{X O}}{s_{x} \sqrt{\left(\frac{1}{n_{A B}}+\frac{1}{n_{B A}}\right)}}
$$

which suggests that $s_{x}$ is a natural standardizer of twice the effect size. Furthermore, since $s_{x}=s_{e} \sqrt{2}$ and $\left(\frac{1}{n_{A B}}+\frac{1}{n_{B A}}\right)=\frac{\left(n_{A B}+n_{B A}\right)}{n_{A B} n_{B A}}$

$$
t=d_{R M} \sqrt{\frac{2 n_{A B} n_{B A}}{\left(n_{A B}+n_{B A}\right)}}
$$

Thus,

$$
\operatorname{var}\left(\delta_{R M}\right)=V(\theta) \frac{\left(n_{A B}+n_{B A}\right)}{2 n_{A B} n_{B A}}
$$

Replacing $\theta$ by $\delta_{R M} \sqrt{\frac{2 n_{A B} n_{B A}}{\left(n_{A B}+n_{B A}\right)}}$ and employing Equation 3:

$$
\operatorname{var}\left(\delta_{R M}\right)=\left(\frac{d f}{d f-2}\right)\left[\frac{\left(n_{A B}+n_{B A}\right)}{2 n_{A B} n_{B A}}+\delta_{R M}^{2}\right]-\frac{\delta_{R M}^{2}}{[c(d f)]^{2}}
$$

This is similar to the equation for the variance of $\frac{\bar{d}_{X} O}{s_{x}}$ given in the Appendix to [1]. The difference is only in the term $2 n_{A B} n_{B A}$ where Curtin et al. use the constant 4 , and we use 2 , because we standardize by $s_{e}$. However, for small sample 
sizes, it is inappropriate to replace $\delta_{R M}$ by $d_{R M}$ in Equation 9, because $d_{R M}$ is biased. For an unbiased estimate of $\delta_{R M}$, we need to use the bias corrected estimate $g_{R M}=c(d f) \times d_{R M}$ giving:

$$
\operatorname{var}\left(d_{R M}\right)=\left(\frac{d f}{d f-2}\right)\left[\frac{\left(n_{A B}+n_{B A}\right)}{2 n_{A B} n_{B A}}+g_{R M}^{2}\right]-\frac{g_{R M}^{2}}{[c(d f)]^{2}}
$$

In addition, multiplying the variance of $d_{R M}$ by $c(d f)^{2}$, an unbiased estimate of the variance of $g_{R M}$ is:

$$
\operatorname{var}\left(g_{R M}\right)=c(d f)^{2}\left(\frac{d f}{d f-2}\right)\left[\frac{\left(n_{A B}+n_{B A}\right)}{2 n_{A B} n_{B A}}+g_{R M}^{2}\right]-g_{R M}^{2}
$$

To construct the variance of $d_{I G}$ it is necessary to consider the relationship between $s_{e}$ and $s_{b}$. This relies on the correlation $\rho$ between values obtained from the same subject:

$$
\rho=\frac{\sigma_{\xi}^{2}}{\sigma_{b}^{2}}=\frac{\sigma_{b}^{2}-\sigma_{e}^{2}}{\sigma_{b}^{2}}
$$

so, $s_{e}=s_{b} \sqrt{(1-\hat{\rho})}$. Thus, based on Equation 1 and Equation 6, the relationship between $t$ and $d_{I G}$ is:

$$
t=d_{I G} \sqrt{\frac{2 n_{A B} n_{B A}}{(1-\hat{\rho})\left(n_{A B}+n_{B A}\right)}}
$$

Therefore replacing $\delta_{R M}$ with $\frac{\delta_{I G}}{\sqrt{(1-\hat{\rho})}}$ in Equation 9 and multiplying by $(1-\hat{\rho})$ gives:

$$
\operatorname{var}\left(\delta_{I G}\right)=\left(\frac{d f}{d f-2}\right)\left[\frac{(1-\hat{\rho})\left(n_{A B}+n_{B A}\right)}{2 n_{A B} n_{B A}}+\delta_{I G}^{2}\right]-\frac{\delta_{I G}^{2}}{[c(d f)]^{2}}
$$

Compared with the Equation 12 in [1], Equation 14 includes the term $(1-\hat{\rho})$ in the first term on the right-hand side of the equation and the term $2 n_{A B} n_{B A}$ rather than $4 n_{A B} n_{B A}$. The inclusion of the term $(1-\hat{\rho})$ in Equation 14 is comparable with the equivalent equation for the variance of pretest-posttest standardized effect size [2].

However, again, if we want the most appropriate variance for small sample sizes, we should not replace $\delta_{I G}$ by $d_{I G}$. Like $d_{R M}, d_{I G}$ is biased for small sample sizes, so we need to replace $\delta_{I G}$ with $g_{I G}=c(d f) \times d_{I G}$ giving:

$$
\operatorname{var}\left(d_{I G}\right)=\left(\frac{d f}{d f-2}\right)\left[\frac{(1-\hat{\rho})\left(n_{A B}+n_{B A}\right)}{2 n_{A B} n_{B A}}+g_{I G}^{2}\right]-\frac{g_{I G}^{2}}{[c(d f)]^{2}}
$$

In addition, the variance of $g_{I G}$ is:

$$
\operatorname{var}\left(g_{I G}\right)=c(d f)^{2}\left(\frac{d f}{d f-2}\right)\left[\frac{(1-\hat{\rho})\left(n_{A B}+n_{B A}\right)}{2 n_{A B} n_{B A}}+g_{I G}^{2}\right]-g_{I G}^{2}
$$

\section{Approximate standardized effect sizes for larger samples}

For an approximate standardized effect size, Curtin et al. [1] use the formula proposed by [5] for $\operatorname{var}\left(d_{R M}\right)$. Since the approximation assumes large sample sizes, the effect of the small sample size adjustment is negligible. So, after correcting a typographical error in [1], the approximate variance for $d_{R M}$ is

$$
\operatorname{var}\left(d_{R M}\right)_{A p p r o x}=\frac{\left(n_{A B}+n_{B A}\right)}{2 n_{A B} n_{B A}}+\frac{d_{R M}^{2}}{2\left(n_{A B}+n_{B A}-3.94\right)}
$$

In addition, based on the relationship between $d_{R M}$ and $d_{I G}$, the large sample size approximation of the variance of $d_{I G}$ is:

$$
\operatorname{var}\left(d_{I G}\right)_{A p p r o x}=(1-\hat{\rho}) \frac{\left(n_{A B}+n_{B A}\right)}{2 n_{A B} n_{B A}}+\frac{d_{I G}^{2}}{2\left(n_{A B}+n_{B A}-3.94\right)}
$$




\section{References}

1. Curtin F, Altman DG, Elbourne D. Meta-analysis combining parallel and cross-over clinical trials. I: Continuous outcomes. Statistics in Medicine 2002; 21:2132-2144, doi:10.1002/sim.1205.

2. Morris SB. Distribution of the standardized mean change effect size for meta-analysis on repeated measures. British Journal of Mathematical and Statistical Psychology 2000; 53:17-29.

3. Morris SB, DeShon RP. Combining effect size estimates in meta-analysis with repeated measures and independent-groups designs. Psychological Methods 2002; 7(1):105-125, doi:10.1037//1082-989X.7.1.105.

4. Johnson NL, Welch BL. Applications of the non-central t-distribution. Biometrika 1940; 31(3/4):362-389.

5. Hedges LV, Olkin I. Statistical methods for meta-analysis. Academic Press: Orlando, Florida, USA, 1985.

6. Senn S. Cross-over Trials in Clinical Research. 2nd edn., John Wiley and Sons, Ltd., 2002. 